\title{
$S$-Carboxymethylcysteine inhibits adherence of Streptococcus pneumoniae to human alveolar epithelial cells
}

\author{
Correspondence \\ Shigetada Kawabata \\ kawabata@dent.osaka-u.ac.jp
}

Received 26 April 2011

Accepted 1 September 2011

\author{
Tomoko Sumitomo, ${ }^{1}$ Masanobu Nakata, ${ }^{1}$ Masaya Yamaguchi, ${ }^{2}$ \\ Yutaka Terao ${ }^{1}$ and Shigetada Kawabata ${ }^{1}$ \\ ${ }^{1}$ Department of Oral and Molecular Microbiology, Osaka University Graduate School of Dentistry, \\ Osaka, Japan \\ ${ }^{2}$ Department of Cell Membrane Biology, Institute of Scientific and Industrial Research, Osaka \\ University, Osaka, Japan
}

\begin{abstract}
Streptococcus pneumoniae is a major pathogen of respiratory infections that utilizes platelet-activating factor receptor (PAFR) for firm adherence to host cells. The mucolytic agent $S$-carboxymethylcysteine ( $S-C M C$ ) has been shown to exert inhibitory effects against infection by several respiratory pathogens including $S$. pneumoniae in vitro and in vivo. Moreover, clinical studies have implicated the benefits of $S-C M C$ in preventing exacerbation of chronic obstructive pulmonary disease, which is considered to be related to respiratory infections. In this study, to assess whether the potency of $S-C M C$ is attributable to inhibition of pneumococcal adherence to host cells, an alveolar epithelial cell line stimulated with interleukin- $1 \alpha$ was used as a model of inflamed epithelial cells. Despite upregulation of PAFR by inflammatory activation, treatment with $S-C M C$ efficiently inhibited pneumococcal adherence to host epithelial cells. In order to gain insight into the inhibitory mechanism, the effects of S-CMC on PAFR expression were also investigated. Following treatment with $S$-CMC, PAFR expression was reduced at both mRNA and post-transcriptional levels. Interestingly, $S$-CMC was also effective in inhibiting pneumococcal adherence to cells transfected with PAFR small interfering RNAs. These results indicate S-CMC as a probable inhibitor targeting numerous epithelial receptors that interact with $S$. pneumoniae.
\end{abstract}

\section{INTRODUCTION}

Streptococcus pneumoniae is a major cause of communityacquired pneumonia, sinusitis and otitis media, and is also associated with acute exacerbations of chronic obstructive pulmonary disease (COPD) (Miravitlles et al., 1999). Among the numerous pneumococcal adherence mechanisms responsible for causing diseases, phosphorylcholine binds specifically to platelet-activating factor receptor (PAFR) expressed on the surface of airway epithelial cells (Cundell et al., 1995, 1996; Fischer, 2000). Previous in vivo experiments demonstrated that PAFR-deficient mice were less likely to develop invasive disease or had improved host defence during pneumococcal infection (Rijneveld et al., 2004). Therefore, the interaction between PAFR and

\footnotetext{
Abbreviations: COPD, chronic obstructive pulmonary disease; DAPI, 4,6-diamidino-2-phenylindole; GSH, glutathione; ICAM-1, intercellular adhesion molecule-1; IL, interleukin; NF- $\kappa \mathrm{B}$, nuclear factor- $\kappa \mathrm{B}$; PAFR, platelet-activating factor receptor; plgR, polymeric immunoglobulin receptor; S-CMC, S-carboxymethylcysteine; siRNA, small interfering RNA.

Supplementary data are available with the online version of this paper.
}

phosphorylcholine plays a crucial role in the pathogenesis of pneumococcal disease.

The mucolytic agent $S$-carboxymethylcysteine (S-CMC) is widely used as an expectorant in Europe and Asia for the treatment of respiratory diseases with phlegm production. Administration of $S$-CMC is also effective for COPD patients in terms of reductions in symptom exacerbation and improvements in quality of life (Zheng et al., 2008). It is generally accepted that bacterial colonization is a determinant for COPD exacerbation. Hence, it has been speculated that the potency of S-CMC against respiratory pathogens, including S. pneumoniae (Cakan et al., 2003; Suer et al., 2008), Moraxella catarrhalis (Zheng et al., 1999) and non-typable Haemophilus influenzae (Ndour et al., 2001), contributes to a reduction in episodes of respiratory infection in COPD patients. However, the mechanism by which $S$-CMC inhibits pneumococcal adherence to host cells is not completely understood.

Several receptors for respiratory pathogens are upregulated in the epithelial cells of patients with COPD (Albelda, 1991; Komatsu et al., 2008; Liu et al., 2008). Thus, it is conceivable that expression of PAFR is also elevated in 
chronically inflamed airways. In fact, activation of airway epithelial cells with inflammatory cytokines results in upregulation of PAFR, which in turn facilitates efficient pneumococcal adherence to epithelial cells (Cundell et al., 1995; Ishizuka et al., 2003). To gain insight into this mechanism, we examined whether $S$-CMC inhibited pneumococcal adherence mediated by phosphorylcholine and PAFR.

\section{METHODS}

Pneumococcal strains and growth conditions. S. pneumoniae serotype 6B strains P-03-90, P-03-160 and P-03-200, and serotype 19F strains P-03-11, P-03-99 and P-03-163, were isolated from sputum samples from patients with pneumonia. The pneumococcal strains were grown in tryptic soy broth (Becton Dickinson) until the lateexponential phase $\left(\mathrm{OD}_{600}\right.$ of 0.6$)$. The cells were then harvested, washed twice with PBS, and resuspended in Dulbecco's modified Eagle's medium (DMEM; Wako) supplemented with $10 \%$ fetal bovine serum (SAFC Biosciences).

Cell culture. The human alveolar carcinoma cell line A549 (RIKEN Cell Bank) derived from type II pneumocytes was maintained in DMEM supplemented with $10 \%$ fetal bovine serum at $37{ }^{\circ} \mathrm{C}$ in a $5 \%$ $\mathrm{CO}_{2}$ atmosphere.

Adherence assay. Adherence assays were performed as described previously (Yamaguchi et al., 2008), with minor modifications. Briefly, A549 cells were cultured in 24-well plates at a density of $2 \times 10^{5}$ cells per well and infected with $2 \times 10^{6}$ c.f.u. bacteria (m.o.i. of 10) for $1 \mathrm{~h}$. For quantification of bacterial adherence, infected cells were washed with PBS and lysed with distilled water. Serial dilutions of the lysates were plated on tryptic soy agar plates to determine the number of c.f.u.

Treatment of epithelial cells with S-CMC. For adherence inhibition assays, A549 cells were treated with various concentrations $\left(100,10,1,0.1,0.01\right.$ and $0.001 \mu \mathrm{g} \mathrm{ml}^{-1}$ ) of $S$-CMC (Kyorin Pharmaceuticals) and incubated at $37{ }^{\circ} \mathrm{C}$ for $30 \mathrm{~min}$ in a $5 \% \mathrm{CO}_{2}$ atmosphere, after which the cells were washed three times with PBS. Control cells were prepared in a similar manner except that they were not treated with S-CMC. The effect of S-CMC on the viability of bacteria and A549 cells was assessed by a plate counting method and trypan blue exclusion assay, respectively.

Treatment of epithelial cells with interleukin (IL)-1 $\alpha$. After the treatment with various concentrations of S-CMC, A549 cells were stimulated with $5 \mathrm{ng}$ IL- $1 \alpha$ (Sigma-Aldrich) $\mathrm{ml}^{-1}$ at $37{ }^{\circ} \mathrm{C}$ for $4 \mathrm{~h}$ in a $5 \% \mathrm{CO}_{2}$ atmosphere, washed three times with PBS and infected with S. pneumoniae.

Immunoblotting. A549 cells were seeded at a concentration of $2 \times 10^{5}$ cells per well (35 mm diameter; Nunc) and cultured for $24 \mathrm{~h}$. After treatment with $S$-CMC, the cells were stimulated with IL-1 $\alpha$. The cells were washed with PBS and then lysed with Laemmli gel loading buffer containing $6 \% 2$-mercaptoethanol, after which the proteins in the cell lysates were separated by SDS-PAGE and blotted onto PVDF membranes. The membranes were blocked with $0.4 \%$ Block Ace solution (DS Pharma Biomedical), followed by incubation with a primary rabbit polyclonal antibody against human PAFR (Cayman) or $\beta$-actin (Cell Signalling) for $1 \mathrm{~h}$ at room temperature. The membranes were then washed with PBS and incubated with horseradish peroxidase-conjugated secondary antibody (Cell Signalling) for $1 \mathrm{~h}$ at room temperature. Following the washing steps, the membranes were developed with ECL Plus Western Blotting Detection Reagent (GE Healthcare). Intensity analysis of the protein bands was performed using Scion Image 4.0.3.2 software (Scion).

Real-time RT-PCR assay. Three independent total RNA samples were isolated from A549 cells using Trizol (Life Technologies) and an RNeasy Mini kit (Qiagen). Synthesis of cDNA from total RNA was conducted with a SuperScript III First-Strand Synthesis System for RT-PCR (Life Technologies). The possibility of DNA contamination was excluded by PCR analysis with non-reverse-transcribed samples. Primer sets for selected genes were designed using Primer Express software (version 3.0; Applied Biosystems). All primers are listed in Supplementary Table S1 (available in JMM Online). RT-PCR was performed using the SYBR Green method with an ABI StepOne Real-Time PCR System (version 2.2; Applied Biosystems). Relative expression rates were calculated by the $\Delta \Delta C_{\mathrm{T}}$ method. The level of glyceraldehyde-3-phosphate dehydrogenase ( $g a p d h)$ expression was used as an internal control.

Immunofluorescence microscopic analysis. A549 cells were seeded at a density of $2 \times 10^{5}$ cells per well onto cover slides pretreated with poly L-lysine (13 mm diameter; Matsunami) and cultured for $24 \mathrm{~h}$ at $37{ }^{\circ} \mathrm{C}$ in a $5 \% \mathrm{CO}_{2}$ atmosphere. The cells were treated with $S$-CMC, followed by stimulation with IL- $1 \alpha$ and then infected with S. pneumoniae strain P-03-11 at an m.o.i. of 10 for $1 \mathrm{~h}$ and fixed with $4 \%$ paraformaldehyde in PBS for $20 \mathrm{~min}$ at room temperature. Finally, the cells were blocked with $5 \%$ BSA in PBS. To observe the localization of $S$. pneumoniae on the epithelial cells, the bacterial cells were visualized with rabbit polyclonal antiserum raised against the serotype 19 capsule (Denka Seiken) and Alexa Fluor 488conjugated anti-rabbit IgG (Molecular Probe). Infected cells were reacted with a primary rabbit polyclonal antibody targeting the human PAFR (Cayman), followed by incubation with Alexa Fluor 594-conjugated anti-rabbit IgG (Molecular Probe). Cell nuclei were stained with 4,6-diamidino-2-phenylindole (DAPI). Finally, the cover slides were mounted onto glass slides using VECTASHIELD mounting medium (Vector) and examined using an LSM 510 confocal microscope system, version 3.2 (Carl Zeiss). Images were obtained and processed with LSM 510 software.

RNA interference. Pre-designed ON-TARGETplus SMARTpool small interfering RNAs (siRNAs) against human PAFR and negative controls were purchased from ThermoScientific and Life Technologies, respectively. A549 cells were transfected with four siRNA duplexes targeting different regions of the PAFR gene at a final concentration of $50 \mathrm{nM}$. Transfection of siRNA was carried out as reported previously (Sumitomo et al., 2011).

Qualitative analysis of capsule production. Analysis of capsule production was carried out by ELISA. S. pneumoniae strains were grown to exponential phase $\left(\mathrm{OD}_{600}=0.4\right)$ and centrifuged at $7000 \mathrm{~g}$ for $5 \mathrm{~min}$. Pelleted cells were resuspended in PBS. Polystyrene plates (Iwaki) were coated with the bacterial suspension and air-dried overnight. The plates were blocked with PBS containing $0.004 \%$ Block Ace, followed by incubation with a primary rabbit polyclonal antibody against the serotype 6 capsule or serotype 19 capsule for $1 \mathrm{~h}$ at room temperature. The plates were then washed with PBS and incubated with horseradish peroxidase-conjugated secondary antibody (Cell Signalling) for $1 \mathrm{~h}$ at room temperature. Following the washing steps, the reaction was developed with TMB peroxidase substrate (Moss). The reactions were stopped with $0.5 \mathrm{M}$ sulfuric acid and the $A_{450}$ was measured.

Statistical analysis. A Kruskal-Wallis test was used to determine whether any of the groups exhibited a statistically significant difference with regard to the percentage of pneumococcal adherence. If the Kruskal-Wallis test results demonstrated at least one of the 
groups to be statistically different, post-hoc analysis using a MannWhitney $U$ test with Bonferroni correction was used to adjust the significance value $(P)$. Simple paired comparisons were performed using a Mann-Whitney $U$ test. A confidence interval with a $P$ value of $<0.05$ was considered to be significant.

\section{RESULTS AND DISCUSSION}

It has been reported that $S$-CMC is effective in preventing pneumococcal adherence to human pharyngeal epithelial cells (Cakan et al., 2003; Suer et al., 2008). As the serotype $6 \mathrm{~B}$ and $19 \mathrm{~F}$ strains have been reported to be major causes of pneumococcal infection throughout the world (Chiba et al., 2005; Henriques-Normark et al., 2008), we first investigated whether S-CMC also inhibited adherence of these types of clinical isolates to the alveolar epithelial cell line A549 (Fig. 1). Utilizing three strains for each serotype and $S$-CMC at concentrations of $0.001-100 \mu \mathrm{g} \mathrm{ml} \mathrm{m}^{-1}$, adherence assays were conducted. S-CMC reduced the adherence of these six strains to A549 cells in a dosedependent manner. In particular, treatment of A549 cells with S-CMC at concentrations of 10 and $100 \mu \mathrm{g} \mathrm{ml}$ resulted in a significant decrease in the adherence of all tested strains compared with untreated cells. In contrast, treatment with $100 \mu \mathrm{g} S$-CMC ml ${ }^{-1}$ did not affect the viability of either the bacteria or the A549 cells (data not shown). The concentration of S-CMC in the blood of healthy adults at $2 \mathrm{~h}$ after a single oral dose of $500 \mathrm{mg}$ is $\sim 5 \mu \mathrm{g} \mathrm{ml}{ }^{-1}$. Thirty years ago, a pharmacokinetic study showed that approximately $4 \mu \mathrm{g} S$-CMC ml ${ }^{-1}$ could be detected in bronchial mucus samples collected from patients with acute exacerbations of chronic bronchitis at $2 \mathrm{~h}$ after a single oral dose of $2 \mathrm{~g}$ S-CMC-Lys, a lysine salt of S-CMC (Braga et al., 1982). Therefore, a level of S-CMC adequate to inhibit pneumococcal adherence on the surface of pharyngeal and alveolar epithelial cells can be expected following such administration.

The capsule is one of the most important pneumococcal virulence factors. Adamou et al. (1998) reported an isogenic acapsulated $S$. pneumoniae strain that adhered to a much greater extent than its encapsulated parental strain to bronchial epithelial cells. Hence, to examine the possibility that capsule production by tested isolates was attributable to differences in their ability to adhere to A549 cells and related to the inhibitory effects of S-CMC,
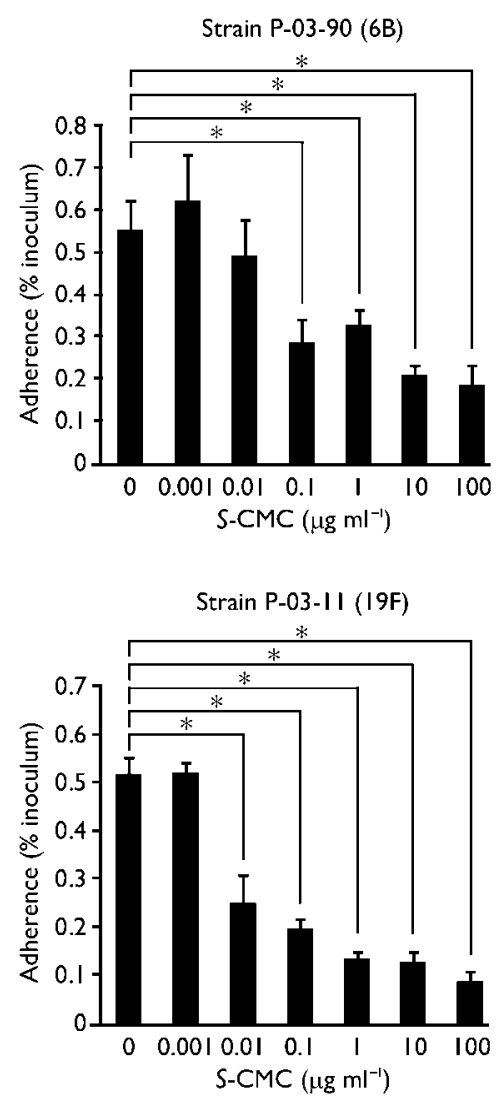

Strain P-03-160 (6B)

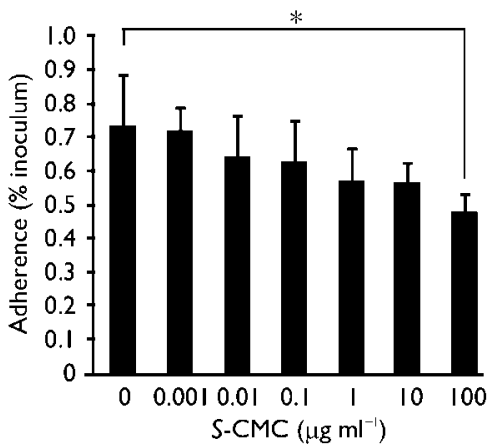

Strain P-03-99 (19F)

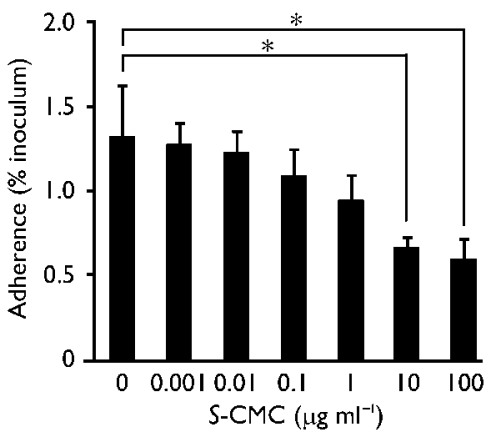

Strain P-03-200 (6B)

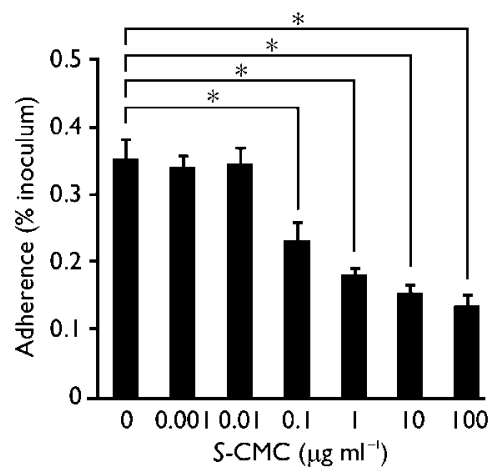

Strain P-03-163 (19F)

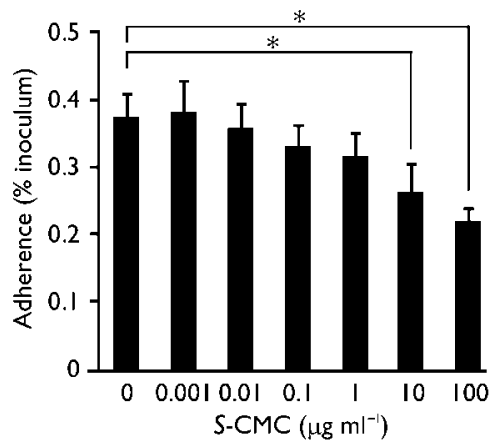

Fig. 1. $S-C M C$ inhibits pneumococcal adherence to human alveolar epithelial cells. A549 cells were treated with $S-C M C$ at $37{ }^{\circ} \mathrm{C}$ for $30 \mathrm{~min}$ and then infected separately with S. pneumoniae serotype 6B strains P-03-90, P-03-160 or P-03-200, and serotype 19F strains P-03-11, P-03-99 or P-03-163, at an m.o.i. of 10 for 1 h. All experiments were performed three times with six technical repeats. Data are presented as means $\pm \mathrm{SD}$ of six wells from a representative experiment. ${ }^{*}, P<0.05$. 
capsule production by each strain was investigated (see Supplementary Fig. S1, available in JMM Online). Although strain P-03-99 had the lowest level of capsule production among the tested strains, it adhered efficiently to the epithelial cells in the absence of S-CMC. However, as no correlation between capsule production and the effect of $S$-CMC on pneumococcal adherence was observed, it is likely that interaction of the pneumococcal capsule with the host receptor is not affected by $S$-CMC.

$S$-CMC has been reported to reduce the number of exacerbations in COPD patients (Zheng et al., 2008). S. pneumoniae is frequently isolated from airway secretions of COPD patients during a period of exacerbation (Sethi \& Murphy, 2001). To examine whether S-CMC inhibits pneumococcal adherence to chronically inflamed host cells, A549 cells stimulated with IL- $1 \alpha$ were subjected to adherence assays. The serotype $6 \mathrm{~B}$ strain P-03-200 and serotype 19F strain P-03-11, which were found to be similar in their level of capsule production, growth rate and adherence ability, were tested in the following experiments. Activation of A549 with IL- $1 \alpha$ caused a fivefold increase in bacterial adherence to the cells in the absence of S-CMC (Fig. 2; compare with Fig. 1), which may have been caused by an increased expression of PAFR that occurs in cells stimulated with IL-1 $\alpha$ (Cundell et al., 1995). Despite the increase in bacterial adherence to the cells, S-CMC efficiently reduced the enhanced adherence of both strains to the epithelial cells in a dose-dependent manner. These results suggest that the potency of $S$-CMC against $S$. pneumoniae adherence contributes to a reduction in COPD symptom exacerbation.

In rhinovirus infections, $S$-CMC reduces expression of the receptor intercellular adhesion molecule-1 (ICAM-1) and production of cytokines in human tracheal epithelial cells (Yasuda et al., 2006). Additionally, S-CMC reduces the concentration of IL-6 in the breath condensate of acute COPD patients (Carpagnano et al., 2004). Based on findings showing that $S$-CMC possesses immunomodulatory properties, we speculated that $S$-CMC inhibits pneumococcal adherence as a result of downregulation of host receptor expression. The interaction of pneumococcal phosphorylcholine with PAFR is critical for the pathogenesis of pneumococcal infections; thus, we examined whether S-CMC repressed the gene expression of PAFR in A549 cells stimulated with IL- $1 \alpha$ (Fig. 3a). Noticeable effects of S-CMC on the expression of PAFR at the transcriptional level were observed in A549 cells stimulated with IL- $1 \alpha$, whilst the amount of ICAM-1 mRNA in A549 cells was also reduced by $S$-CMC treatment. Non-typable $H$. influenzae is also an important pathogen of COPD exacerbation (Veeramachaneni \& Sethi, 2006) and binds to a variety of host receptors, including PAFR and ICAM-1 (Swords et al., 2000, 2001; Avadhanula et al., 2006). It has been suggested that S-CMC-induced modulation of cellsurface charge contributes to a reduction in adherence of $H$. influenzae to epithelial cells. The results of our real-time PCR analysis raised the possibility that S-CMC could
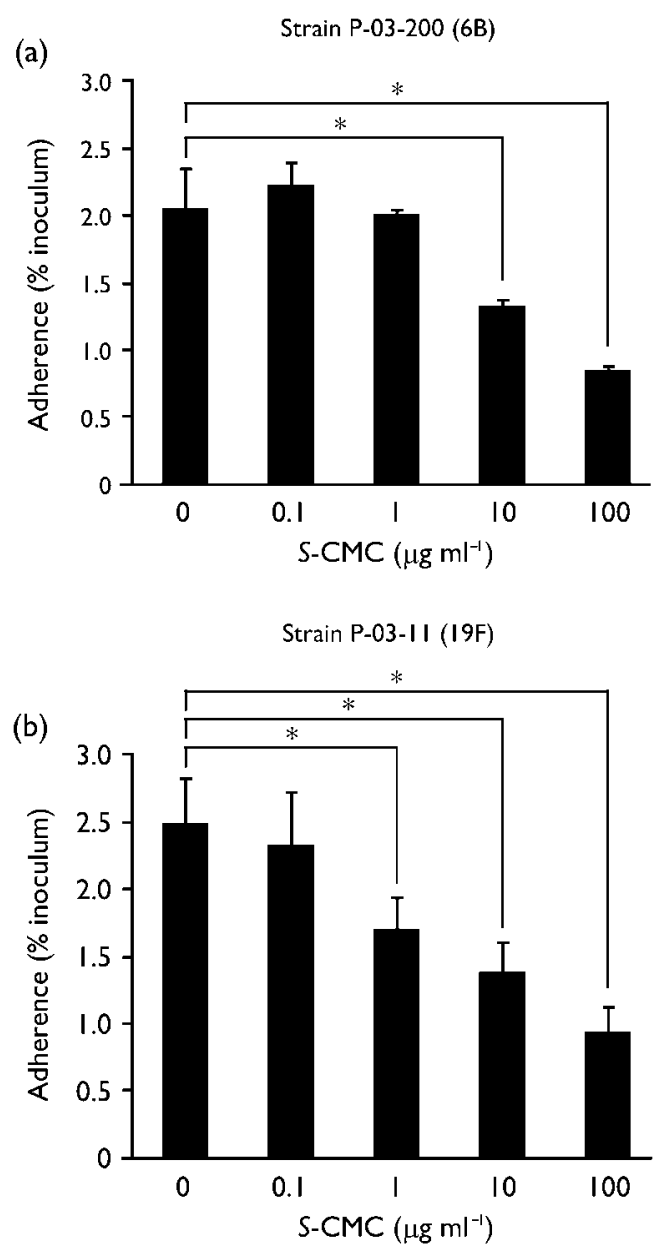

Fig. 2. $S$-CMC inhibits pneumococcal adherence to IL-1 $\alpha$-treated human alveolar epithelial cells. A549 cells were treated with $S$ $\mathrm{CMC}$ at $37{ }^{\circ} \mathrm{C}$ for $30 \mathrm{~min}$, followed by activation with $5 \mathrm{ng} \mathrm{IL}-1 \alpha$ $\mathrm{ml}^{-1}$ for $4 \mathrm{~h}$. Thereafter, the cells were infected with $S$. pneumoniae strain P-03-200 (a) or P-03-11 (b) at an m.o.i. of 10 for $1 \mathrm{~h}$. Three experiments were performed and data are presented as means $\pm S D$ of six wells from a representative experiment. * $P<0.05$.

inhibit the adherence of $H$. influenzae to host epithelial cells by reducing the expression of PAFR and ICAM-1, thereby reducing exacerbation rates in COPD patients. The effects of S-CMC on the expression of PAFR in A549 cells stimulated with IL- $1 \alpha$ were assessed by immunoblot analysis (Fig. 3b). Treatment of A549 cells with IL- $1 \alpha$ enhanced the expression of PAFR in the absence of $S$ CMC. Although $\beta$-actin was not affected by treatment with $S$-CMC, PAFR expression was significantly inhibited by increased concentrations of $S$-CMC at the post-transcriptional level. Next, to examine whether $S$-CMC inhibited the interaction of S. pneumoniae and A549 cells stimulated with IL- $1 \alpha$, pneumococcal co-localization with PAFR was investigated using immunofluorescence microscopy (Fig. 3c). Adhered bacteria were observed on cells without 


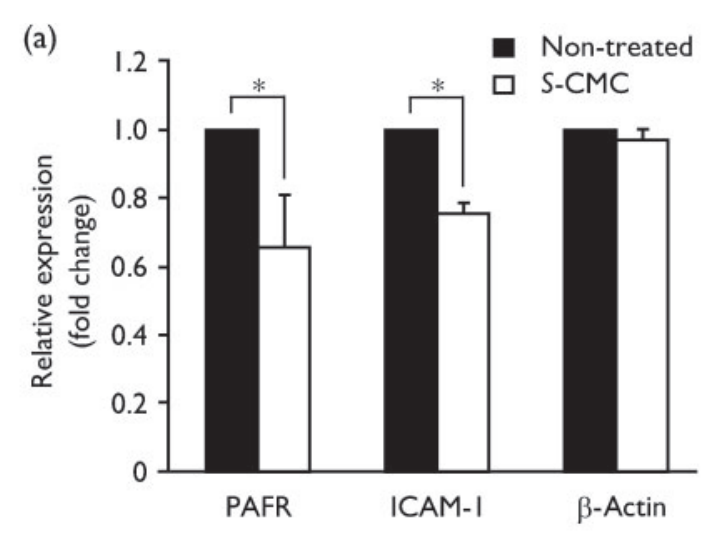

(c)
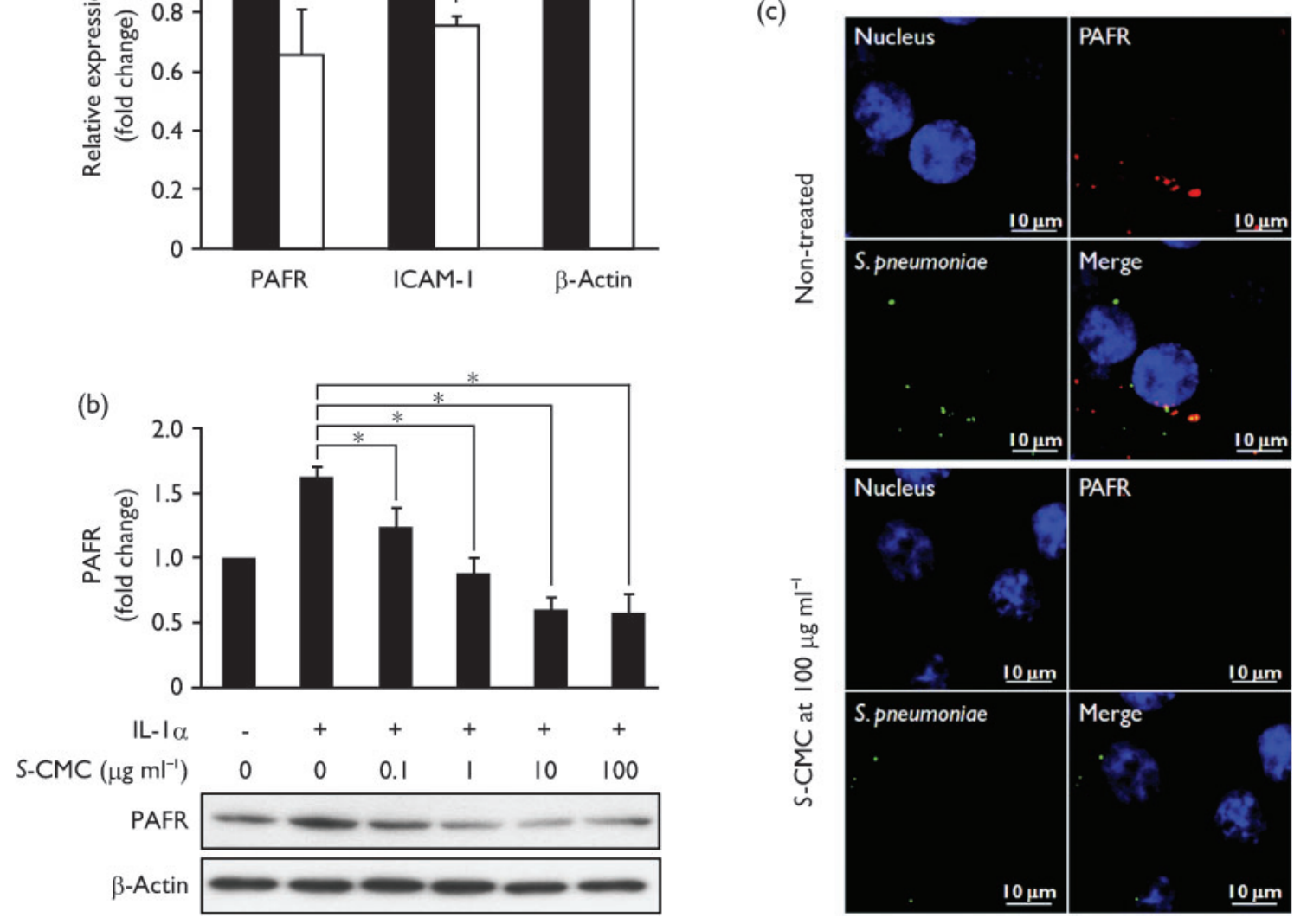

Fig. 3. $S-C M C$ reduces the expression of PAFR. (a) A549 cells were treated with $S-C M C$ at $37{ }^{\circ} \mathrm{C}$ for 30 min and then stimulated with $5 \mathrm{ng} \mathrm{IL}-1 \alpha \mathrm{ml}^{-1}$ for $4 \mathrm{~h}$. cDNA was prepared from the cells and subjected to real-time RT-PCR analysis. The graph shows fold transcript level changes of PAFR, ICAM-1 and $\beta$-actin in cells treated with S-CMC (open bars) relative to non-treated cells (filled bars). Three independent experiments were performed and data are presented as means $\pm S D$ of six samples from a representative experiment. ${ }^{*}, P<0.01$. (b) A549 cells were treated with $S-C M C$ at $37{ }^{\circ} \mathrm{C}$ for 30 min and then stimulated with $5 \mathrm{ng} \mathrm{IL}-1 \alpha \mathrm{ml}^{-1}$ for 4 h. PAFR expression was detected in whole-cell lysates by immunoblot analysis. $\beta$-Actin served as a loading control. The graph shows quantification of the PAFR bands shown in the blots. The intensity of the bands was digitized by Scion Image alpha 4.0.3.2 software (Scion) and normalized using that of $\beta$-actin, and representative results are presented as means \pm SD of three independent experiments. Data are presented as fold expression levels relative to cells not treated with $S-C M C$ and IL-1 $\alpha .{ }^{*}, P<0.05$. (c) A549 cells were treated with $S-C M C$ at $100 \mu g \mathrm{ml}^{-1}$ for 30 min and then stimulated with IL-1 $\alpha$ at $5 \mathrm{ng} \mathrm{ml}^{-1}$ for $30 \mathrm{~min}$. Thereafter, the cells were infected separately with S. pneumoniae strain P-03-11 at an m.o.i. of 10 for 1 h. S. pneumoniae was visualized using an anti-capsule (serotype 19) and Alexa Fluor 488-conjugated antibodies. PAFR was stained with the anti-PAFR and Alexa Fluor 594-conjugated antibodies. DAPI was used to stain the DNA in the nucleus.

$S$-CMC treatment, some of which were co-localized with PAFR on epithelial cells. In contrast, there was a marked loss of fluorescence intensity for PAFR and adhered bacteria in $S$-CMC-treated cells. Furthermore, co-localization of bacteria with PAFR was scarcely observed in cells treated with $S$ CMC compared with non-treated cells. Taken together, our results demonstrated that $S$-CMC reduces the expression of PAFR, which in turn may inhibit the interaction of $S$. pneumoniae with host epithelial cells. To investigate further the mechanism by which $S$-CMC inhibits pneumococcal adherence via PAFR and phosphorylcholine, expression of PAFR was silenced by siRNAs, and PAFR was effectively knocked down by transfection of siRNAs into A549 cells (Fig. 4). The ability of S. pneumoniae to adhere to A549 cells transfected with PAFR siRNA was decreased compared with cells transfected with scrambled control oligonucleotides, indicating that PAFR is a major receptor on the surface of A549 cells for both $S$. pneumoniae strains. As expected, $S$-CMC inhibited the adherence of S. pneumoniae to A549 cells transfected with scrambled control oligonucleotides. 
(a)

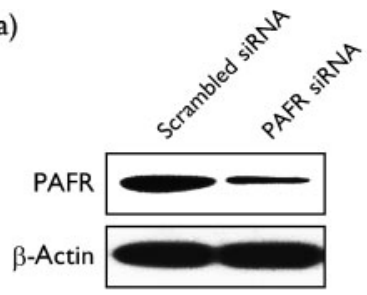

(b)
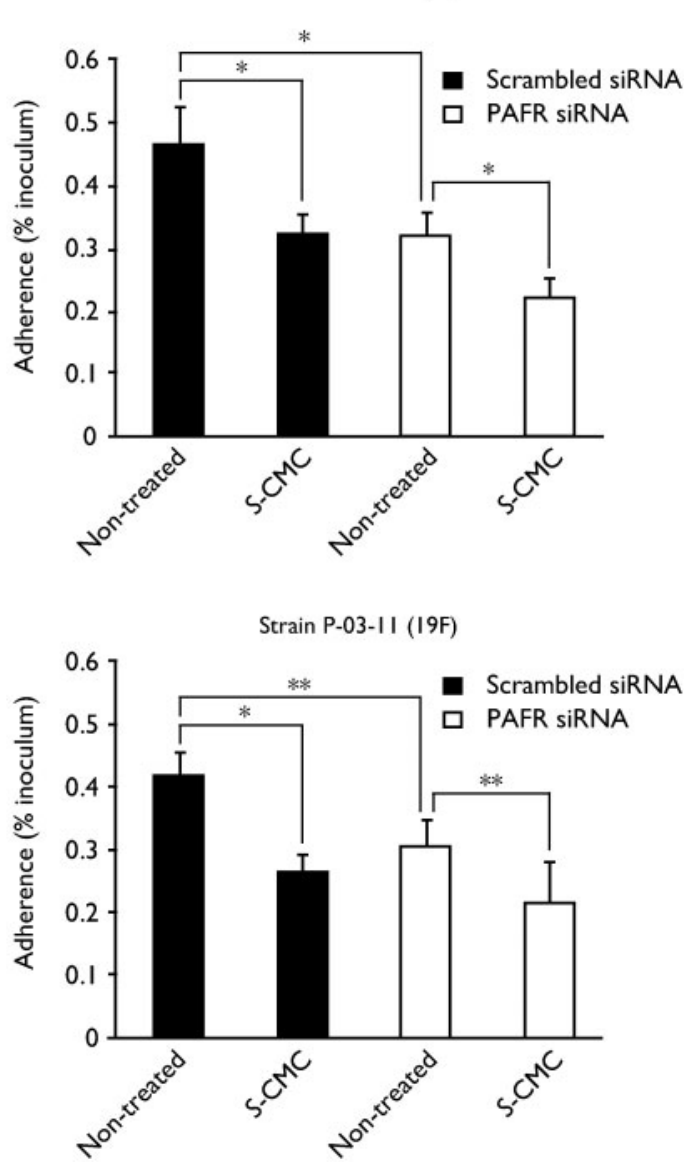

Fig. 4. $S-C M C$ inhibits pneumococcal adherence to PAFR knockdown cells. (a) A549 cells were transfected with a scrambled control or PAFR-targeted siRNA. At $24 \mathrm{~h}$ after transfection, whole-cell lysates were subjected to Western blot analysis using anti-PAFR antibodies. $\beta$-Actin served as a loading control. (b) A549 cells were transfected with PAFR-targeted siRNA. A scrambled siRNA was utilized as a negative control. At $24 \mathrm{~h}$ after transfection, cells were treated with $100 \mu \mathrm{g} \mathrm{S}$-CMC $\mathrm{ml}^{-1}$ at $37^{\circ} \mathrm{C}$ for $30 \mathrm{~min}$, followed by infection with $S$. pneumoniae strain P-03-200 or P-03-11 at an m.o.i. of 10 for $1 \mathrm{~h}$. All experiments were performed six times with three technical repeats. Data are presented as the means $\pm S D$ of six wells from a representative experiment. ${ }^{*}, P<0.01 ;{ }^{\star \star}, P<0.05$.
Although S-CMC unexpectedly inhibited pneumococcal adherence to A549 cells transfected with PAFR siRNA, this may have been due to the inhibitory effect of $S$-CMC on residual PAFR not knocked down in those cells. In addition, our findings raise the possibility that $S$-CMC represses several pneumococcal receptors, such as laminin receptor (Orihuela et al., 2009) and cytokeratin 10 (Shivshankar et al., 2009), on the surface of host cells in addition to PAFR.

Oxidative stress is an important feature in the pathogenesis of COPD. Increases in levels of reactive oxygen species have been implicated in initiating inflammatory responses in the lungs through activation of the transcriptional factor nuclear factor- $\kappa \mathrm{B}(\mathrm{NF}-\kappa \mathrm{B})$ (Rahman, 2006). The production of IL- $1 \alpha$ and tumour necrosis factor- $\alpha$ is also induced by NF- $\kappa \mathrm{B}$ activation, which in turn facilitates PAFR expression (Mutoh et al., 1994). S-CMC-Lys has an antioxidant action that leads to a significant increase of glutathione (GSH) flux through the cell membrane (Guizzardi et al., 2006; Garavaglia et al., 2008). Such an increase in intracellular GSH induces a reduction in inflammatory cytokine release through inhibition of NF$\kappa \mathrm{B}$ activation (Aoki et al., 1996; Antonicelli et al., 2002; Biswas \& Rahman, 2009). A previous study reported that the pyrrolidine derivative pyrrolidine dithiocarbamate, an NF$\kappa \mathrm{B}$ inhibitor, reduces acid-induced $S$. pneumoniae adherence to A549 cells via inhibition of PAFR induction (Ishizuka et al., 2001). Together, these findings suggest that the reduction in PAFR expression in epithelial cells treated with $S$-CMC is attributable to suppression of NF- $\kappa \mathrm{B}$ activation by GSH flux. Polymeric immunoglobulin receptor (pIgR), a pneumococcal receptor of human epithelial cells, is also positively regulated by NF- $\kappa \mathrm{B}$ (Bruno \& Kaetzel, 2005). However, epithelial cells from the lower respiratory tract, such as A549 cells, express only traces of pIgR (Zhang et al., 2000). As pIgR is expressed in the upper respiratory tract at a high level, S-CMC may also have an effect on pneumococcal adherence via pIgR on the surface of these epithelial cells.

In $M$. catarrhalis infections, it has been demonstrated that decreased bacterial adherence in the presence of $S$-CMC is related to the ability of $S$-CMC to deplete the carbohydrate structure on the surface of pharyngeal epithelial cells (Zheng et al., 1999). Another study noted that S. pneumoniae probably recognizes a protein determinant irrespective of the presence or location of the carbohydrate on PAFR (García Rodríguez et al., 1995). Based on our results, we cannot exclude the possibility that $S$-CMC also affects the carbohydrate structure of PAFR.

The present results demonstrated that $S$-CMC inhibits $S$. pneumoniae adherence to alveolar epithelial cells via downregulation of PAFR. As numerous respiratory pathogens, including Neisseria meningitidis and Pseudomonas aeruginosa, express phosphorylcholine on their surface to bind PAFR (Weiser et al., 1998), we speculate that S-CMC is extensively effective for respiratory tract infections caused by these pathogens. 
In conclusion, to our knowledge this is the first report to show that $S$-CMC inhibits pneumococcal adherence to alveolar epithelial cells by reducing the expression of host receptors for S. pneumoniae, including PAFR. Our findings provide new insight into the underlying mechanism of the effectiveness of S-CMC for prevention of $S$. pneumoniae infection and may lead to novel therapeutic applications for respiratory infections.

\section{ACKNOWLEDGEMENTS}

We would like to thank Kyorin Pharmaceutical Co. Ltd, Japan, for financial support for this study. We thank K. Oishi for providing the S. pneumoniae strains and M. Higashino for helpful technical assistance. This work was supported by a grant-in-aid for Scientific Research on young scientists (B) from the Ministry of Education, Culture, Sports, Science and Technology (MEXT) (no. 20791336) awarded to T.S., a grant-in-aid for scientific research on priority areas from MEXT (no. 18073011) awarded to S.K., a grant-in-aid for scientific research (B) from the Japan Society for the Promotion of Science (JSPS) (no. 20390465) awarded to S. K., a grant-in-aid for Scientific Research on young scientists (A) from MEXT (no. 2189048) awarded to Y. T. and a grant-in-aid for Scientific Research on young scientists (B) from MEXT (no. 19791343) awarded to M.N.

\section{REFERENCES}

Adamou, J. E., Wizemann, T. M., Barren, P. \& Langermann, S. (1998), Adherence of Streptococcus pneumoniae to human bronchial epithelial cells (BEAS-2B). Infect Immun 66, 820-822.

Albelda, S. M. (1991). Endothelial and epithelial cell adhesion molecules. Am J Respir Cell Mol Biol 4, 195-203.

Antonicelli, F., Parmentier, M., Drost, E. M., Hirani, N., Rahman, I., Donaldson, K. \& MacNee, W. (2002). Nacystelyn inhibits oxidantmediated interleukin-8 expression and NF- $\kappa \mathrm{B}$ nuclear binding in alveolar epithelial cells. Free Radic Biol Med 32, 492-502.

Aoki, T., Suzuki, Y., Suzuki, K., Miyata, A., Oyamada, Y., Takasugi, T., Mori, M., Fujita, H. \& Yamaguchi, K. (1996). Modulation of ICAM-1 expression by extracellular glutathione in hyperoxia-exposed human pulmonary artery endothelial cells. Am J Respir Cell Mol Biol 15, 319_ 327.

Avadhanula, V., Rodriguez, C. A., Ulett, G. C., Bakaletz, L. O. \& Adderson, E. E. (2006). Nontypeable Haemophilus influenzae adheres to intercellular adhesion molecule 1 (ICAM-1) on respiratory epithelial cells and upregulates ICAM-1 expression. Infect Immun 74, 830-838.

Biswas, S. K. \& Rahman, I. (2009). Environmental toxicity, redox signaling and lung inflammation: the role of glutathione. Mol Aspects Med 30, 60-76.

Braga, P. C., Borsa, M., De Angelis, L., Bossi, R., Allegra, L., Scaglione, F. \& Scarpazza, G. (1982). Pharmacokinetic behavior of $S$-carboxymethylcysteine-Lys in patients with chronic bronchitis. Clin Ther 4, 480-488.

Bruno, M. E. \& Kaetzel, C. S. (2005). Long-term exposure of the HT-29 human intestinal epithelial cell line to TNF causes sustained up-regulation of the polymeric Ig receptor and proinflammatory genes through transcriptional and posttranscriptional mechanisms. J Immunol 174, 7278-7284.

Cakan, G., Turkoz, M., Turan, T., Ahmed, K. \& Nagatake, T. (2003). $S$-carboxymethylcysteine inhibits the attachment of Streptococcus pneumoniae to human pharyngeal epithelial cells. Microb Pathog 34, 261-265.

Carpagnano, G. E., Resta, O., Foschino-Barbaro, M. P., Spanevello, A., Stefano, A., Di Gioia, G., Serviddio, G. \& Gramiccioni, E. (2004). Exhaled Interleukine-6 and 8-isoprostane in chronic obstructive pulmonary disease: effect of carbocysteine lysine salt monohydrate (SCMC-Lys). Eur J Pharmacol 505, 169-175.

Chiba, N., Kobayashi, R., Hasegawa, K., Morozumi, M., Nakayama, E., Tajima, T., Iwata, S., Ubukata, K. \& Acute Respiratory Diseases Study Group (2005). Antibiotic susceptibility according to genotype of penicillin-binding protein and macrolide resistance genes, and serotype of Streptococcus pneumoniae isolates from community-acquired pneumonia in children. J Antimicrob Chemother 56, 756-760.

Cundell, D. R., Gerard, N. P., Gerard, C., Idanpaan-Heikkila, I. \& Tuomanen, E. I. (1995). Streptococcus pneumoniae anchor to activated human cells by the receptor for platelet-activating factor. Nature 377, 435-438.

Cundell, D. R., Gerard, C., Idanpaan-Heikkila, I., Tuomanen, E. I. \& Gerard, N. P. (1996). PAf receptor anchors Streptococcus pneumoniae to activated human endothelial cells. Adv Exp Med Biol 416, 89-94.

Fischer, W. (2000). Phosphocholine of pneumococcal teichoic acids: role in bacterial physiology and pneumococcal infection. Res Microbiol 151, 421-427.

Garavaglia, M. L., Bononi, E., Dossena, S., Mondini, A., Bazzini, C., Lanata, L., Balsamo, R., Bagnasco, M., Conese, M. \& other authors (2008). S-CMC-Lys protective effects on human respiratory cells during oxidative stress. Cell Physiol Biochem 22, 455-464.

García Rodríguez, C., Cundell, D. R., Tuomanen, E. I., Kolakowski, L. F., Jr, Gerard, C. \& Gerard, N. P. (1995). The role of N-glycosylation for functional expression of the human platelet-activating factor receptor. Glycosylation is required for efficient membrane trafficking. J Biol Chem 270, 25178-25184.

Guizzardi, F., Rodighiero, S., Binelli, A., Saino, S., Bononi, E., Dossena, S., Garavaglia, M. L., Bazzini, C., Bottà, G. \& other authors (2006). S-CMC-Lys-dependent stimulation of electrogenic glutathione secretion by human respiratory epithelium. J Mol Med (Berl) 84, 97-107.

Henriques-Normark, B., Blomberg, C., Dagerhamn, J., Bättig, P. \& Normark, S. (2008). The rise and fall of bacterial clones: Streptococcus pneumoniae. Nat Rev Microbiol 6, 827-837.

Ishizuka, S., Yamaya, M., Suzuki, T., Nakayama, K., Kamanaka, M., Ida, S., Sekizawa, K. \& Sasaki, H. (2001). Acid exposure stimulates the adherence of Streptococcus pneumoniae to cultured human airway epithelial cells: effects on platelet-activating factor receptor expression. Am J Respir Cell Mol Biol 24, 459-468.

Ishizuka, S., Yamaya, M., Suzuki, T., Takahashi, H., Ida, S., Sasaki, T., Inoue, D., Sekizawa, K., Nishimura, H. \& Sasaki, H. (2003). Effects of rhinovirus infection on the adherence of Streptococcus pneumoniae to cultured human airway epithelial cells. J Infect Dis 188, 19281939.

Komatsu, K., Jono, H., Lim, J. H., Imasato, A., Xu, H., Kai, H., Yan, C. \& Li, J.-D. (2008). Glucocorticoids inhibit nontypeable Haemophilus influenzae-induced MUC5AC mucin expression via MAPK phosphatase-1-dependent inhibition of p38 MAPK. Biochem Biophys Res Commun 377, 763-768.

Liu, K., Gualano, R. C., Hibbs, M. L., Anderson, G. P. \& Bozinovski, S. (2008). Epidermal growth factor receptor signaling to Erk1/2 and STATs control the intensity of the epithelial inflammatory responses to rhinovirus infection. J Biol Chem 283, 9977-9985.

Miravitlles, M., Espinosa, C., Fernández-Laso, E., Martos, J. A., Maldonado, J. A., Gallego, M. \& Study Group of Bacterial Infection in COPD (1999). Relationship between bacterial flora in sputum and 
functional impairment in patients with acute exacerbations of COPD. Chest 116, 40-46.

Mutoh, H., Ishii, S., Izumi, T., Kato, S. \& Shimizu, T. (1994). Plateletactivating factor (PAF) positively auto-regulates the expression of human PAF receptor transcript 1 (leukocyte-type) through NF- $\kappa \mathrm{B}$. Biochem Biophys Res Commun 205, 1137-1142.

Ndour, C. T., Ahmed, K., Nakagawa, T., Nakano, Y., Ichinose, A., Tarhan, G., Aikawa, M. \& Nagatake, T. (2001). Modulating effects of mucoregulating drugs on the attachment of Haemophilus influenzae. Microb Pathog 30, 121-127.

Orihuela, C. J., Mahdavi, J., Thornton, J., Mann, B., Wooldridge, K. G., Abouseada, N., Oldfield, N. J., Self, T., Ala'Aldeen, D. A. \& Tuomanen, E. I. (2009). Laminin receptor initiates bacterial contact with the blood brain barrier in experimental meningitis models. J Clin Invest 119, 1638-1646.

Rahman, I. (2006). Antioxidant therapies in COPD. Int J Chron Obstruct Pulmon Dis 1, 15-29.

Rijneveld, A. W., Weijer, S., Florquin, S., Speelman, P., Shimizu, T., Ishii, S. \& van der Poll, T. (2004). Improved host defense against pneumococcal pneumonia in platelet-activating factor receptordeficient mice. J Infect Dis 189, 711-716.

Sethi, S. \& Murphy, T. F. (2001). Bacterial infection in chronic obstructive pulmonary disease in 2000: a state-of-the-art review. Clin Microbiol Rev 14, 336-363.

Shivshankar, P., Sanchez, C., Rose, L. F. \& Orihuela, C. J. (2009). The Streptococcus pneumoniae adhesin PsrP binds to Keratin 10 on lung cells. Mol Microbiol 73, 663-679.

Suer, E., Sayrac, S., Sarinay, E., Ozturk, H. E., Turkoz, M., Ichinose, A., Nagatake, T. \& Ahmed, K. (2008). Variation in the attachment of Streptococcus pneumoniae to human pharyngeal epithelial cells after treatment with S-carboxymethylcysteine. J Infect Chemother 14, 333336.

Sumitomo, T., Nakata, M., Higashino, M., Jin, Y., Terao, Y., Fujinaga, Y. \& Kawabata, S. (2011). Streptolysin $S$ contributes to group A streptococcal translocation across an epithelial barrier. J Biol Chem 286, 2750-2761.
Swords, W. E., Buscher, B. A., Ver Steeg li, K., Preston, A., Nichols, W. A., Weiser, J. N., Gibson, B. W. \& Apicella, M. A. (2000). Nontypeable Haemophilus influenzae adhere to and invade human bronchial epithelial cells via an interaction of lipooligosaccharide with the PAF receptor. Mol Microbiol 37, 13-27.

Swords, W. E., Ketterer, M. R., Shao, J., Campbell, C. A., Weiser, J. N. \& Apicella, M. A. (2001). Binding of the non-typeable Haemophilus influenzae lipooligosaccharide to the PAF receptor initiates host cell signalling. Cell Microbiol 3, 525-536.

Veeramachaneni, S. B. \& Sethi, S. (2006). Pathogenesis of bacterial exacerbations of COPD. COPD 3, 109-115.

Weiser, J. N., Goldberg, J. B., Pan, N., Wilson, L. \& Virji, M. (1998). The phosphorylcholine epitope undergoes phase variation on a 43-kilodalton protein in Pseudomonas aeruginosa and on pili of Neisseria meningitidis and Neisseria gonorrhoeae. Infect Immun 66, 4263-4267.

Yamaguchi, M., Terao, Y., Mori, Y., Hamada, S. \& Kawabata, S. (2008). PfbA, a novel plasmin- and fibronectin-binding protein of Streptococcus pneumoniae, contributes to fibronectin-dependent adhesion and antiphagocytosis. J Biol Chem 283, 36272-36279.

Yasuda, H., Yamaya, M., Sasaki, T., Inoue, D., Nakayama, K., Yamada, M., Asada, M., Yoshida, M., Suzuki, T. \& other authors (2006). Carbocisteine inhibits rhinovirus infection in human tracheal epithelial cells. Eur Respir J 28, 51-58.

Zhang, J. R., Mostov, K. E., Lamm, M. E., Nanno, M., Shimida, S., Ohwaki, M. \& Tuomanen, E. (2000). The polymeric immunoglobulin receptor translocates pneumococci across human nasopharyngeal epithelial cells. Cell 102, 827-837.

Zheng, C. H., Ahmed, K., Rikitomi, N., Martinez, G. \& Nagatake, T. (1999). The effects of $S$-carboxymethylcysteine and $\mathrm{N}$-acetylcysteine on the adherence of Moraxella catarrhalis to human pharyngeal epithelial cells. Microbiol Immunol 43, 107-113.

Zheng, J.-P., Kang, J., Huang, S.-G., Chen, P., Yao, W.-Z., Yang, L., Bai, C.-X., Wang, C.-Z., Wang, C. \& other authors (2008). Effect of carbocisteine on acute exacerbation of chronic obstructive pulmonary disease (PEACE Study): a randomised placebo-controlled study. Lancet 371, 2013-2018. 